\title{
Operation for recurrent ventricular tachycardia
}

\author{
Predictors of short- and long-term efficacy
}

The success of ventricular operation in ablating drug-refractory ventricular tachycardia secondary to ischemic heart disease varies with surgical technique, the presence of certain identified risk factors, and patient selection biases. Forty-eight patients with drug-refractory ventricular tachycardia secondary to ischemic heart disease underwent directed ventricular operation. All patients had previous myocardial infarction, and 46 of 48 patients had a left-ventricular aneurysm. Mapping was done in $81 \%$ of patients. Patients underwent a combination of subendocardial resection, aneurysmectomy, and cryoablation. The operative mortality rate was $8 \%$. Age greater than 65 years was the only risk factor for operative mortality. Forty-one patients underwent postoperative programmed electrical stimulation. In 26 patients $(63 \%)$ tachycardia was noninducible, whereas it was inducible in 15 patients $(37 \%)$. Stepwise logistic regression identified septal and inferior focus location as the most significant predictors of outcome. Septal focus location was a significant $(p=0.008)$ predictor of surgical success whereas inferior focus location was a significant $(p=0.015)$ predictor of surgical failure. Other identified independent risk factors for surgical failure were (1) use of cardioplegia, (2) lack of a completed intraoperative endocardial map, and (3) decreased ejection fraction. This generated model to predict success or failure had a sensitivity of $93.3 \%$ and a specificity of $92.4 \%$. The success of ventricular operation is affected by the presence of certain risk factors. In the management of those patients at high risk for failure, other surgical options such as the placement of implantable cardioverter-defibrillator electrode patches at the time of ventricular operation or the alternative placement of a palliative implantable cardioverter-defibrillator should be considered. (J THORAC CARDIOVASC SURG 1994;107:732-42)

Raymond Lee, MD, John D. Mitchell, MD, Hasan Garan, MD, Jeremy N. Ruskin, MD, Brian A. McGovern, MD, Mortimer J. Buckley, MD, David F. Torchiana, MD, and

Gus J. Vlahakes, MD, Boston, Mass.

Pro rogrammed electrical stimulation (PES) testing provides an objective means of selecting an appropriate antiarrhythmic drug regimen for patients with recurrent ventricular tachycardia (VT). Patients with recurrent VT whose arrhythmias are rendered noninducible by PESguided therapy remain at low risk of recurrent VT and sudden cardiac death. ${ }^{1-3}$ However, in as many as $50 \%$ to

From the Departments of Surgery (Cardiac Surgical Unit) and Medicine (Cardiac Unit), Massachusetts General Hospital and Harvard Medical School, Boston, Mass.

Received for publication April 9, 1993.

Accepted for publication July 7, 1993.

Address for reprints: Gus J. Vlahakes, MD, Department of Surgery, Massachusetts General Hospital, Boston, MA 02114-2696.

Copyright $\odot 1994$ by Mosby-Year Book, Inc.

$0022-5223 / 94 \$ 3.00+0 \quad 12 / 1 / 50715$

$70 \%$ of all patients with recurrent VT secondary to ischemic heart disease, inducible VT persists despite multiple antiarrhythmic drug trials. As a result, this group of patients must rely on invasive options to decrease the risk of recurrent VT by means of (1) ablation of VT with directed ventricular operation or percutaneous radiofrequency ablation or (2) palliation of VT with an implantable cardioverter-defibrillator (ICD).

Since Couch's report ${ }^{4}$ of the first successful surgical ablation of VT after an aneurysmectomy in 1958, operation for VT has advanced dramatically. Current approaches to VT operation use directed subendocardial resection (SER), various methods of intraoperative mapping, and cryosurgery either alone or as a supplement to SER. The overall results of these directed approaches to VT operation are notable for an operative mortality rate that ranges from $5 \%$ to $23 \%$ and a success rate as 
predicted by postoperative PES that ranges from $62 \%$ to 98\%. ${ }^{5-10}$ The variations in operative mortality and surgical success rates are undoubtedly related not only to institutional differences in surgical technique, but also to substantial differences in patient selection. In this study we retrospectively examined our experience with 48 consecutive patients with medically refractory VT secondary to ischemic heart disease who have had ventricular operation and attempted to identify clinical, anatomic, and electrophysiologic characteristics that are correlates of short- and long-term efficacy.

\section{Materials and methods}

Patient population. The study group comprised 48 consecutive patients with documented recurrent, sustained VT who underwent ventricular operation for VT at the Massachusetts General Hospital between January 1984 and December 1991. In all cases the patients were intolerant to, or the VT was refractory to, antiarrhythmic drug therapy. All patients were offered directed ventricular operation unless they had very poor systolic function of the nonaneurysmal ventricular segments with an excess ejection fraction less than $10 \%$ as previously described. ${ }^{5}$ These patients with global cardiomyopathic ventricles were offered ICD therapy because of their much higher operative mortality with directed ventricular operation. There were 41 men and 7 women with an average age of $61 \pm 8$ years (range 43 to 76). All patients underwent catheterization of the right and left sides of the heart, coronary angiography, and left ventriculography. Ejection fraction was calculated by contrast or radionuclide ventriculography.

Preoperative electrophysiologic testing. Preoperative PES testing and catheter mapping were done with electrode catheters $(1 \mathrm{~cm}$ interpolar distance) at multiple left and right ventricular recording sites. These areas were selected according to a standardized mapping grid shown in Fig. 1 that comprised 24 different recording sites, each with an area of approximately 3 by $3 \mathrm{~cm}$. Catheter position during mapping was determined by multiplane fluoroscopy. VT was initiated by PES with single, double, and triple premature extrastimuli at two different cycle lengths from two different sites. The site of origin of reentrant VT was defined as the recording site that demonstrated the earliest presystolic local electrogram late in diastole. " VTs in the same patient were considered different if they manifested different surface electrocardiographic bundle branch configurations and if their mean frontal and horizontal plane axes differed by more than 45 degrees.

Operation. After cannulation for cardiopulmonary bypass, epicardial reference electrodes were sutured to the anterior right and left lateral ventricles for ventricular pacing and reference electrogram recording, respectively. After the institution of normothermic cardiopulmonary bypass, VT was induced by programmed cardiac stimulation. After VT induction, either a single hand-held bipolar electrode ( $2 \mathrm{~mm}$ interpolar distance) or a multielectrode sock ( 64 bipolar electrodes, Bard Cardiopulmonary Division, Tewksbury, Mass.) or both instruments, was used to generate an epicardial activation sequence map. After epicardial mapping was complete, a ventriculotomy was then done through the aneurysm or ventricular scar during VT. If VT terminated after ventriculotomy, PES was used to reinduce VT.
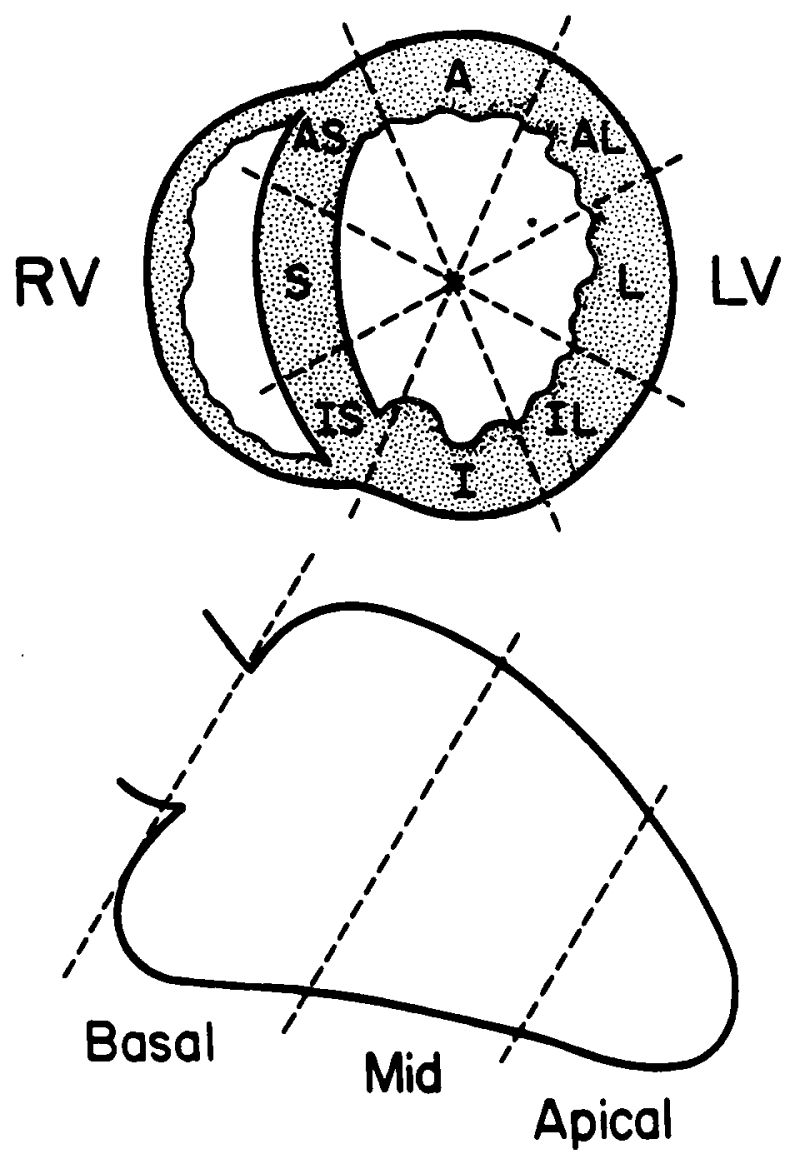

Fig. 1. Preoperative catheter mapping grid that identifies 24 different recording sites, each with area of approximately 4 by $4 \mathrm{~cm}$. Recording sites are located circumferentially around transverse section of heart (top) at different planes perpendicular to long axis of left ventricle ( $L V$ ) from apex to base (bottom). $A$, Anterior; $A L$, anterolateral; $A S$, anteroseptal; $I$, inferior; $I L$, inferolateral; $I S$, inferoseptal; $L$, lateral; $R V$, right ventricle; $S$, - septal. Reprinted with permission from the American College of Cardiology (J Am Coll Cardiol 1986;8:201-9.5)

Endocardial activation sequence mapping was then done with a fingertip electrode ( $2 \mathrm{~mm}$ interpolar distance) with use of the technique of sequential single-site recording normalized to the reference electrogram. After endocardial mapping was complete, an aneurysmectomy was done followed by a wide regional 2 to $3 \mathrm{~mm}$ deep SER of visible endocardial scar in the region of the mapped site of origin of the VT. In several cases of inferior wall infarction, particularly those in which the inferobasal wall was involved but the apex was spared, an area of inferior akinesis was identified but no area of frank dyskinesis was noted; in these cases, the area was only incised rather than being subjected to resection followed by SER. Visible endocardial scar in areas remote from the mapped origin was not removed. The SER was considered limited if the size of the endocardial patch resected was smaller than one recording site $(3$ by $3 \mathrm{~cm}$ ) because of the small extent of visible endocardial scar or because of proximity to the membranous septum, aortic or mitral valve 
anuli, or the base of a papillary muscle. Supplemental cryoablation was then done to the mapped origin, which included the periphery of the SER, with varying numbers of cryoprobe applications at $-60^{\circ} \mathrm{C}$ or below for 2 minutes per application (Frigitronics, Inc., Shelton, Conn.). If coronary artery bypass grafting or valve replacement was required, the patient was then cooled, the aorta was crossclamped, and cardioplegia was administered. If VT could not be induced and mapped in the operating room, a wide regional SER and cryoablation were done at a site identified according to an algorithm based on surface electrocardiogram (ECG) and ventricular pace mapping as previously described. ${ }^{12}$ Other procedures such as coronary artery bypass grafting or valve replacement were then performed. The aortic crossclamp was removed, and the ventriculotomy was closed. After aneurysmectomy, anteroapical and inferoapical aneurysms were closed with exclusion of the aneurysm with nonabsorbable interrupted sutures with Teflon felt strip reinforcement to support the ventriculotomy closure. Patients were then weaned from cardiopulmonary bypass.

Postoperative evaluation. All patients were continuously monitored for cardiac rhythm after the operation. Postoperative PES testing was done approximately 10 to 14 days after operation. The initiation of VT was attempted using single, double, and triple premature extrastimuli at two different cycle lengths from two different sites including the site of VT origin as determined by preoperative or intraoperative mapping. Patients who continued to have inducible VT by PES after operation then underwent serial drug testing in attempts to suppress the induced VT. Those patients who had inducible VT despite ventricular operation and drug therapy then underwent ICD implantation.

Follow-up. Long-term follow-up for this group of patients was concluded in May 1992. Patients were followed up through serial visits to the cardiac arrhythmia unit at the Massachusetts General Hospital or through correspondence with referring cardiologists and with telephone interviews with patients. Patients with late recurrent $\mathrm{VT}$ after hospital discharge received ICD therapy. The functional status of each patient was described using the New York Heart Association classification.

Statistical evaluation. Values for continuous data are presented as mean plus or minus the standard deviation. Stepwise logistic regression analysis by the BMDP statistical software package (BMDP Statistical Software, Inc., Los Angeles, Calif.) was used to identify independent risk factors for the postoperative presence of inducible VT and for operative mortality. Analysis of the site of origin of VT as a risk factor for the postoperative presence of inducible VT was limited to those patients having an activation sequence map. Factors used in the stepwise logistic regression analysis for the postoperative presence of inducible VT and operative mortality were as follows: age; location of myocardial infarction (MI); time since MI; presence and location of a left ventricular aneurysm; New York Heart Association heart failure class; extent of coronary artery disease; left ventricular end-diastolic pressure; cardiac index; ejection fraction; previous cardiac operation; need for preoperative intraaortic balloon counterpulsation; preoperative use, duration, and time since discontinuation of amiodarone; electrocardiographic morphology; rate of VT; number of VT episodes; number of ventricular fibrillation episodes; number of VT morphologies; number and location of identified sites of VT origin; completion of preoperative catheter map, intraoperative epicardial map, and/or intraoperative endocardial map; cardiopul- monary bypass time; aortic crossclamp time; use of cardioplegia; use of cryoablation; performance and extent of coronary artery bypass grafting; valve replacement; performance and extent of endocardial resection; aneurysmectomy; location of ventriculotomy; and placement of ICD defibrillator patch electrodes. Actuarial analysis was used to generate probability of survival and freedom from recurrent VT data.

Definitions. Sustained monomorphic VT was defined as VT with uniform QRS complexes, persistent for at least 30 seconds or requiring cardioversion because of hemodynamic compromise; operative mortality indicated death within 30 days after operation or before hospital discharge; nonclinical VT was defined as VT induced during postoperative PES testing that was not previously observed; operation-alone success indicated no spontaneous postoperative VT during hospital stay and no inducible VT at postoperative PES testing without any antiarrhythmic medications; operation-medication success indicated no spontaneous postoperative VT during hospital stay or inducible VT at postoperative PES testing after the addition of an antiarrhythmic regimen; late recurrent $V T$ indicated any VT occurring after hospital discharge; and clinical success was defined as no late recurrent VT during the follow-up period with or without antiarrhythmic medication.

\section{Results}

Patient population. All 48 patients studied had a history of a previous MI (anterior 54\%, inferior 36\%, both $10 \%$ ) with 11 patients undergoing operation within 2 months of infarction. All but two patients had angiographic evidence of a left ventricular aneurysm. Cardiac catheterization revealed a mean left ventricular enddiastolic pressure of $17 \pm 8 \mathrm{~mm} \mathrm{Hg}$. The mean left ventricular ejection fraction was $32 \% \pm 12 . \%$. Thirty-six patients $(73 \%)$ had significant stenosis in at least two coronary arteries with five patients having severe left main coronary stenosis. Preoperative intraaortic balloon counterpulsation was required in three patients for hemodynamic support or control of ischemia. In 46 patients serial drug trials failed, with an average of $3.7 \pm 1.6$ failed drugs per patient. Two patients did not undergo serial drug testing; both had multiple spontaneous VT episodes and were not studied because of severe coronary artery disease and hemodynamic instability. Amiodarone had been given to 20 patients before operation with an average duration of not receiving amiodarone before operation of $10 \pm 13$ weeks. These and other patient characteristics are shown in Table I.

Preoperative PES testing. PES testing was completed in 41 of 48 patients. Seven patients did not undergo complete PES testing because of severe left main coronary artery disease in four patients, a ventricular pseudoaneurysm in one patient, hemodynamically unstable VT in one patient, and unreliable VT induction in the presence of residual amiodarone despite multiple spontaneous VT episodes in one patient. In the 41 patients with completed 
Table I. Patient characteristics (total patient population, $\mathrm{N}=48$ )

\begin{tabular}{|c|c|c|}
\hline & $\begin{array}{c}\text { No. of } \\
\text { patients }\end{array}$ & $\%$ \\
\hline Age $(y r)$ & $61 \pm 8$ (range $43-76$ ) & \\
\hline \multicolumn{3}{|l|}{ Sex } \\
\hline Male & 41 & 85 \\
\hline Female & 7 & 15 \\
\hline MI & 48 & 100 \\
\hline Anterior MI & 26 & 54 \\
\hline Inferior MI & 17 & 36 \\
\hline Both & 5 & 10 \\
\hline $\begin{array}{l}\text { Time from MI to operation } \\
(\mathrm{mo})\end{array}$ & $52 \pm 86$ & \\
\hline$\leq 2$ months & 11 & 23 \\
\hline$>2$ months & 37 & 77 \\
\hline \multicolumn{3}{|l|}{ NYHA class } \\
\hline I & 17 & 35 \\
\hline II & 11 & 23 \\
\hline III & 14 & 29 \\
\hline IV & 6 & 13 \\
\hline $\begin{array}{l}\text { Previous cardiac } \\
\text { operation }\end{array}$ & 2 & 5 \\
\hline Episodes VT (per pt.) & $4.3 \pm 3.5($ range $0-16)$ & \\
\hline $\begin{array}{l}\text { No. of cardioversions } \\
\text { (per pt.) }\end{array}$ & $2.3 \pm 3.5($ range $0-23)$ & \\
\hline Ejection fraction (\%) & $32 \pm 12$ (range $10-59)$ & \\
\hline LVEDP (mm Hg) & $17 \pm 8$ & \\
\hline $\begin{array}{l}\text { Cardiac index } \\
\left(\mathrm{L} / \mathrm{min} / \mathrm{m}^{2}\right)\end{array}$ & $2.6 \pm 0.5$ & \\
\hline Failed drugs (per pt.) & $3.7 \pm 1.6$ & \\
\hline Amiodarone & 20 & 42 \\
\hline
\end{tabular}

$N Y H A$, New York Heart Association; $p t$, patient; $L V E D P$, left ventricular enddiastolic pressure.

electrophysiologic studies, there were 61 distinct VTs induced for an average of $1.5 \pm 0.8$ surface ECG morphologies per patient. There were 26 patients $(63 \%)$ with only a single VT surface ECG configuration and 15 patients $(37 \%)$ with multiple sustained monomorphic VTs with distinct ECG morphologies. The average rate of the induced VT was $196 \pm 44$ beats/min with 18 patients having rates greater than 200 beats/min. Right ventricular pacing was successful in terminating induced VT in 29 of the 41 patients $(71 \%)$. These electrophysiologic data are summarized in Table II.

VT mapping. Preoperative transcatheter endocardial activation sequence mapping was completed in $27(56 \%)$ of 48 patients. Preoperative activation sequence mapping was not attempted in the remaining 21 patients because of significant left main coronary artery disease, pseudoaneurysm, or hemodynamic instability with induced VT necessitating immediate termination.

Complete epicardial activation sequence maps were done in the operating room in 34 patients. In the remain-
Table II. Electrophysiologic data (total patient population, $\mathrm{N}=48$ )

\begin{tabular}{lc}
\hline $\begin{array}{c}\text { Completed preoperative PES (No. of } \\
\text { patients) }\end{array}$ & 41 \\
Total No. of induced VTs & 61 \\
Single morphology (No. of patients) & 26 \\
Multiple morphologies (No. of patients) & 15 \\
No. of morphologies (per patient) & $1.5 \pm 0.8$ (range 0-4) \\
Morphology & $37(61 \%)$ \\
RBBB (No. of VTs) & $24(39 \%)$ \\
LBBB (No. of VTs) & $196 \pm 44$ \\
VT rate (beats/min) & $29(71 \%)$ \\
Conversion & $12(29 \%)$ \\
RV pacing (No. of patients) & \\
Cardioversion (No. of patients) &
\end{tabular}

$R B B B$, right bundle branch block; $L B B B$, left bundle branch block; $R V$, right ventricular.

ing patients, epicardial maps could not be done because of absence of inducible VT in the operating room despite its presence preoperatively, rapid VT rate precluding detailed mapping, VT that degenerated into ventricular fibrillation, or a left ventricular pseudoaneurysm. Complete intraoperative endocardial mapping was done in nine patients. In 25 patients with completed epicardial mapping, intraoperative endocardial mapping was not possible because of the inability to induce sustained clinical VT after ventriculotomy.

Overall, 39 patients ( $81 \%$ ) had some type of mapping procedure completed. Twenty-nine patients $(71 \%)$ had a complete epicardial activation sequence map done and 29 patients $(60 \%)$ had an endocardial activation sequence map (either preoperative or intraoperative) completed. In nine patients ( $19 \%)$ no activation sequence mapping during VT could be completed and the surgical resection was guided by the ECG algorithm and pace mapping. These data are shown in Table III.

The results of the activation sequence mapping procedures completed in 39 patients revealed a total of 59 foci with an average of $1.5 \pm 0.7$ foci per patient. Twentyfour patients were found to have only a single site of origin, whereas 15 patients were discovered to have multiple disparate sites of origin. The locations of the mapped foci are shown in Table IV. As shown, anterior foci are related to anterior MI. Septal foci are related to both anterior and inferior MIs and are comprised of those foci in midseptal, anteroseptal, and inferoseptal locations according to the mapping grid shown in Fig. 1. For purposes of data analysis, the septal, anteroseptal, and inferoseptal locations have been grouped together in what will hereafter be referred to as septal foci. Inferior foci are also related to both anterior and inferior MI. The inferior foci associat- 
Table III. Mapping procedures*

\begin{tabular}{cccc}
\hline $\begin{array}{c}\text { No. of } \\
\text { patients }\end{array}$ & $\begin{array}{c}\text { Preoperative } \\
\text { catheter map }\end{array}$ & $\begin{array}{c}\text { Intraoperative } \\
\text { epicardial map }\end{array}$ & $\begin{array}{c}\text { Intraoperative } \\
\text { endocardial map }\end{array}$ \\
\hline 7 & + & + & + \\
15 & + & + & - \\
10 & - & + & - \\
5 & + & - & - \\
2 & - & + & +
\end{tabular}

*No. of patients with any mapping: $39(81 \%)$; No. of patients without any mapping: $9(19 \%)$; No. of patients with any endocardial map: $29(60 \%)$; No. of patients with only epicardial map: $10(21 \%)$; percents given are percent of total.

ed with anterior infarction were all in the inferoapical region and associated with a large occluded "wraparound" apical left anterior descending coronary artery.

Operation. Table $\mathrm{V}$ outlines the various procedures performed on 48 patients. Aneurysmectomy was done in $90 \%$ of patients. A wide regional subendocardial resection was possible and completed in $79 \%$ of patients, and cryoablation was done in $73 \%$ of patients. Twenty-seven patients $(56 \%)$ received a combination of a wide SER, cryosurgery, and aneurysmectomy. In nine patients a wide regional SER was not possible because of either the small extent of visible endocardial scarring or proximity of endocardial scar to membranous septum, aortic or mitral valve anuli, or posterior papillary muscle.

There were two deaths within 30 days of operation and an additional two deaths that occurred later than 30 days after operation but before hospital discharge, for a total operative mortality of four patients $(8 \%)$. The causes of death in these four cases were sepsis, stroke, aortic dissection, and low-output congestive heart failure. None of the patients with operative death had evidence of recurrent VT after operation. After operation, five patients required intraaortic balloon counterpulsation for hemodynamic support in addition to the three patients who had intraaortic balloons placed preoperatively. Reoperation for mitral valve replacement was necessary in three patients within 2 weeks of ventricular operation because of significant mitral regurgitation. All three of these patients had an inferoseptal focus and had a wide regional inferoseptal SER with supplemental cryosurgery around the base of the posterior papillary muscle.

Postoperative PES. Forty-one of the 44 operative survivors underwent postoperative PES testing. Twentysix patients had neither spontaneous nor PES-induced VT in the absence of antiarrhythmic drugs for an operationalone success rate of $63 \%$. Fifteen patients had inducible VT at postoperative PES testing. Of these 15 patients, 4 patients had a nonclinical VT induced, which was not
Table IV. Activation sequence mapping data*

\begin{tabular}{lccc}
\hline & \multicolumn{2}{c}{ No. of patients with $M I$} \\
\cline { 3 - 4 } Location of foci & No. of patients & Anterior MI & Inferior MI \\
\hline Anterior & 14 & 13 & 1 \\
Septal & 19 & 12 & 7 \\
Lateral & 1 & 0 & 1 \\
Inferior & 19 & 9 & 10 \\
Right ventricle & 3 & 2 & 1 \\
\hline
\end{tabular}

*Total No. of mapped foci: 59 ; No. of patients with single focus: 24 ; No. of patients with multiple foci: 15 .

possible to reproduce. The remaining 11 patients had an induced VT that had been documented previously. Of the 15 patients with postoperative inducible VT, five had the VT suppressed and subsequently had no inducible VT when given antiarrhythmic drugs that had previously failed to control the tachycardia in three patients. Therefore the combined operation-medication success rate was $76 \%$ (31 patients).

Follow-up. The patient follow-up for the study was $100 \%$. The average follow-up time was $41 \pm 27$ months with a range of 3 to 96 months. Twelve patients were followed up beyond 5 years. A flow chart that illustrates the operative results is shown in Fig. 2. Among the 26 patients who had no postoperative PES-induced VT without medications, there was only one late VT recurrence, which occurred at 5 months ( $96 \%$ clinical success rate). The three patients who required reoperation for mitral valve replacement had noninducible VT at postoperative PES and did not have any late VT recurrence. Among the five patients who initially had an inducible VT at postoperative PES but none when taking antiarrhythmic drugs, there were two late VT recurrences. However, one of these recurrences occurred in a patient in whom the clinical VT was induced at postoperative PES and was then successfully suppressed by procainamide, which previously had been unsuccessful. VT did not recur for 56 months until a lupus syndrome developed in the patient; the procainamide was then stopped with prompt VT recurrence. Overall, among the 31 patients who had no inducible VT with or without medications before hospital discharge, there were only three late VT recurrences ( $90 \%$ clinical success rate).

Among the 15 patients who had inducible VT at postoperative PES, there were a total of six late VT recurrences (60\% clinical success rate). Among these 15 patients, the four patients who manifested nonclinical VT at postoperative PES had no recurrences. All six of the late recurrences occurred in the remaining 11 patients with inducible postoperative clinical VT $(45 \%$ clinical 
Table V. Operations performed

\begin{tabular}{|c|c|c|c|c|}
\hline No. of patients & Wide regional $S E R$ & Limited SER & Cryosurgery & Aneurysmectomy \\
\hline 27 & + & - & + & + \\
\hline 2 & + & - & + & - \\
\hline 8 & + & - & - & + \\
\hline 2 & - & + & + & + \\
\hline 2 & - & + & - & + \\
\hline 1 & - & + & + & - \\
\hline 2 & - & - & + & + \\
\hline$\overline{1}$ & - & - & + & - \\
\hline 2 & - & - & - & + \\
\hline Wide regional SER (No. of patients) & \multicolumn{4}{|c|}{38} \\
\hline Limited SER (No. of patients) & \multicolumn{4}{|c|}{5} \\
\hline Cryosurgery (No. of patients) & \multicolumn{4}{|c|}{35} \\
\hline Aneurysmectomy (No. of patients) & \multicolumn{4}{|c|}{43} \\
\hline CABG (No. of patients) & \multicolumn{4}{|c|}{34} \\
\hline Mean No. of grafts (per patient) & \multicolumn{4}{|c|}{$3.1 \pm 1.6$} \\
\hline CPB time (min) & \multicolumn{4}{|c|}{$150 \pm 54$} \\
\hline Aortic crossclamp (No. of patients) & \multicolumn{4}{|c|}{35} \\
\hline Mean crossclamp time (min) & \multicolumn{4}{|c|}{$63 \pm 24$} \\
\hline
\end{tabular}

$C A B G$, Coronary artery bypass grafting; $C P B$, cardiopulmonary bypass.

success rate). Of the seven patients who continued to have inducible VT despite antiarrhythmic drug therapy, four patients had late VT recurrence and received ICD therapy.

Overall, there were seven cases of late VT recurrence among the 44 operative survivors for a clinical success rate of $84 \%$. In six of the seven late VT recurrences, the VT morphology was identical to the preoperative morphology. An inferior focus was identified in three of the seven late VT recurrences. There was only one septal focus identified in the patients with late VT recurrence, but this was identified in a patient who also had an inferior focus. The actuarial freedom from recurrent VT at 1,3 , and 5 years was $88.5 \%, 84.7 \%$, and $77.9 \%$, respectively (Fig. 3).

There were five late deaths in the 44 operative survivors; none were attributed to sudden cardiac death or documented arrhythmia. The causes of death were stroke after brain operation, pneumonia, MI, and progressive congestive heart failure. Two of the three patients who required reoperative mitral valve replacement are included in the late deaths; one patient died at 24 months after operation of influenza pneumonia, and the other patient died 10 months after operation of an MI. The actuarial survival rate not including the four operative deaths at 1 , 3 , and 5 years was $97.6 \%, 94.1 \%$, and $82.49 \%$, respectively. Including the operative deaths, the actuarial survival rate at 1,3 , and 5 years was $89.5 \%, 86.3 \%$, and 75.5\%, respectively (Fig. 4).

Predictors of operative mortality. Stepwise logistic regression analysis identified age 65 years or greater as the only independent risk factor $(p=0.031)$ for operative mortality.

Predictors of surgical success. Stepwise logistic regression analysis identified the location of the site of VT origin to be the strongest independent factor predictive of postoperative PES results. The presence of a septal focus was a significant $(p=0.008)$ predictor of postoperative PES noninducibility; that is, patients with septal foci were less likely to have their VT inducible at postoperative PES than patients whose foci were located elsewhere. Conversely, the presence of an inferior focus was a significant $(p=0.015)$ predictor of persistent postoperative PES-induced VT. In addition to the site of origin, further logistic regression analysis identified the following factors in order of their relative significance (most statistically significant items appearing first) to be independent predictors of postoperative PES inducibility: (1) use of cardioplegia $(p=0.027)$, (2) lack of an intraoperative endocardial map $(p=0.045)$, and (3) decreased ejection fraction $(p=0.011)$. The use of cardioplegia, the lack of an intraoperative endocardial map, and the presence of an inferior site of VT origin were all independent positive predictors of postoperative PES inducibility. The five factors identified by stepwise logistic regression were used to create a model that can predict postoperative PES inducibility. When this model is applied to the patient population of 41 patients who had a postoperative PES, the model demonstrates a sensitivity of $93.3 \%$ and a specificity of $92.4 \%$. 


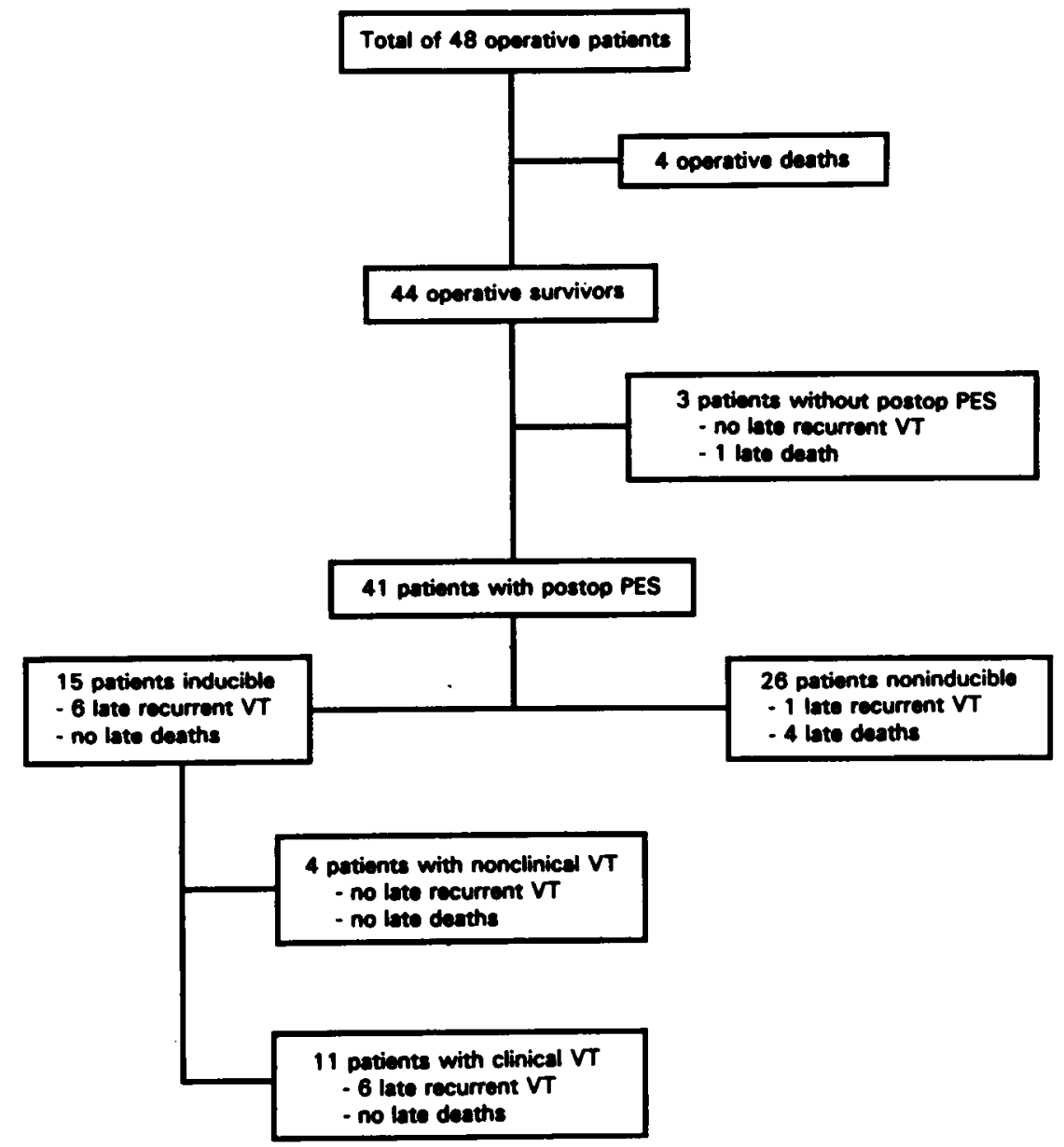

Fig. 2. Flowchart of operative results. Postop, Postoperative.

\section{Discussion}

This study demonstrates that directed ventricular operation can provide patients with medically refractory VT a chance for electrophysiologic cure with an $8 \%$ operative mortality rate. The efficacy of operation has been shown to be predicted reliably by the postoperative PES study. In this study only $3(10 \%)$ of the 31 patients with a negative postoperative PES study had late VT recurrence compared with $4(40 \%)$ of the 10 patients with a positive postoperative PES study $(p=0.027)$. The operation-alone success rate of $63 \%$ and the clinical success rate of $84 \%$ in this study are comparable to the experience reported by other institutions. ${ }^{13-18}$ Despite differences in the surgical treatment of VT, other institutions have identified several common factors associated with a higher risk of surgical failure.

The specific location of the site of origin appears to be the most common risk factor for surgical failure to cure VT identified by various reported series. When focus location data are reported, the prevalence of an inferior focus varies from $21 \%$ to $52 \%$ of patients in the various reported series..$^{13-18}$ The prevalence of inferior foci in our study was comparable, with $49 \%$ of the patients who underwent activation sequence mapping having an inferior focus. The reason for the increased risk of surgical failure may be attributed to the greater difficulty in exposing the inferior endocardial surface of the heart and completing an endocardial map, as well as the greater difficulty in performing a wide regional SER because of the presence of the posterior papillary muscle and the often thin inferior ventricular wall. Patients with inferior left ventricular aneurysms were also more likely to have had a more limited left ventricular aneurysmectomy, compared with anterior and apical left ventricular aneurysms. The results in patients having an inferior focus may be improved by the use of supplemental cryoablation in addition to SER in the area bounded by the mitral anulus and the base of the posterior papillary muscle. ${ }^{13}$ In all 


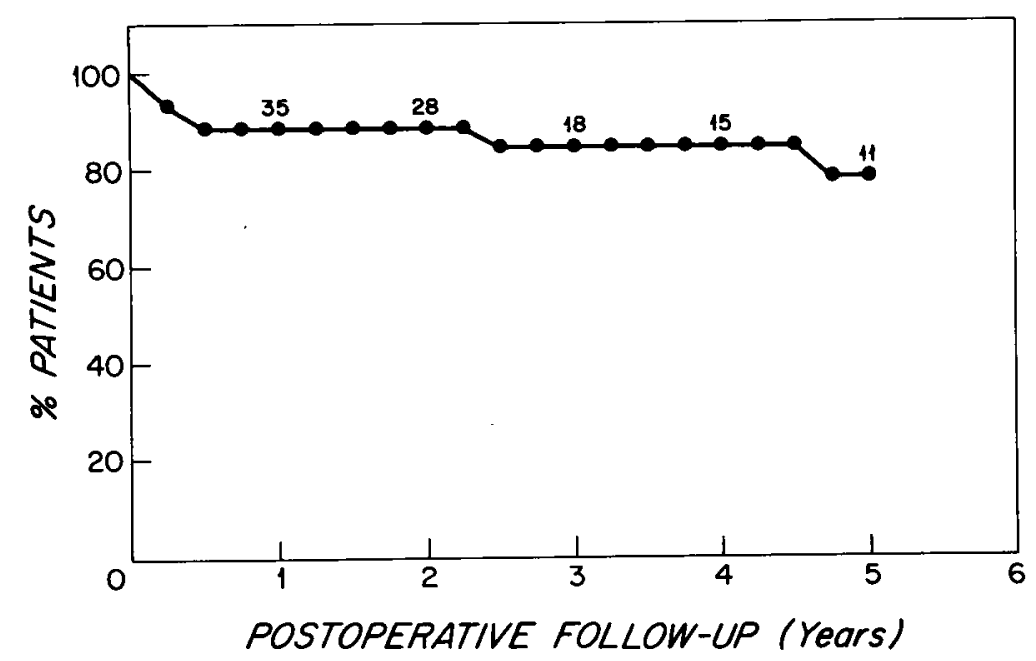

Fig. 3. Actuarial freedom from recurrent VT (percent) of all 44 operative survivors during 5-year postoperative follow-up period.

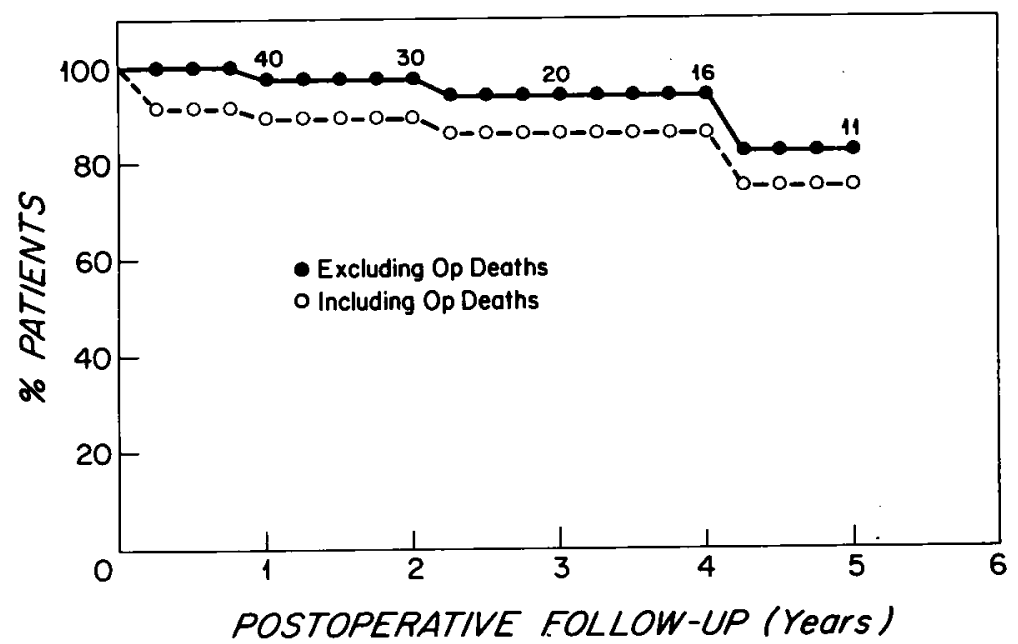

Fig. 4. Actuarial survival (percent) of patients during 5-year postoperative follow-up period. Op, Operative.

of the patients with inferior foci reported on in this study, supplemental cryoablation was used in addition to SER in the areas of the mapped focus. Though some groups have advocated routine resection of the papillary muscle and mitral valve replacement, ${ }^{19}$ making a wide SER easier to do in this location, we have not adopted this practice because of the associated increases in perioperative morbidity and mortality and the commitment to chronic anticoagulation with mechanical valve replacement. In this report, patients having an inferior focus had a much lower operation-alone success rate of only $55 \%$ compared with an operation-alone success rate of $83 \%$ if all patients with an inferior focus had been eliminated from the study.
Additionally, of the seven patients who had late recurrent VT, three patients had an identified inferior focus.

Despite the risks associated with an inferior focus, our study identified the presence of a septal site of origin as a significant predictor of surgical success rather than failure. Among the seven cases of late recurrent VT, three patients had an inferior focus whereas only one patient had an identified septal focus. In fact, the patient with the septal focus also had a second inferior focus. The foci that we have grouped into the septal category in this study comprise foci located in the anteroseptal, septal, and inferoseptal regions of the mapping grid shown in Fig. 1. These patients were grouped in this way because of the 
uniform surgical success observed in all three subgroups. The improved surgical success rate in septal VTs has been attributed to the easier exposure afforded by the anterior ventriculotomy. However, in our study, the increased surgical success of septal VTs was independent of the ventriculotomy site. Sixty-three percent of patients with a septal focus had an anterior MI and underwent anterior ventriculotomy and aneurysmectomy, whereas $37 \%$ had an inferior MI and at operation underwent inferior ventriculotomy with a more limited aneurysmectomy in most cases. Alternate explanations are that both anterior and inferior approaches to the septum provide excellent operative exposure of this region of the endocardium or that VT arising from septal foci, whether secondary to an anterior or inferior MI, have a reentrant substrate that may be more amenable to ablation by subendocardial resection and cryoablation compared with VTs arising from the inferior left ventricular free wall.

Two previous studies have evaluated patients with medically refractory VT after an inferior MI and have concluded that these patients pose no greater risk of surgical failure. ${ }^{7,20}$ These studies, however, do not report data on the location of the mapped site of origin. A detailed analysis of the site of arrhythmogenesis may better define the risk of surgical failure posed by inferior MI. Our results show that there is a subset of patients with an inferior MI that does well if the VT has an inferoseptal site of origin, whereas the remainder of the patients with an inferior MI remain at higher risk for surgical failure because of an inferior or inferolateral focus on the left ventricular free wall. Identification of the foci location appears to be a more accurate predictor of surgical failure in patients with an inferior MI.

The lack of an intraoperative endocardial map was identified as a risk factor for postoperative PES-induced VT. None of the nine patients who had an intraoperative endocardial map completed had inducible VT at postoperative PES and none had a late VT recurrence. The presence of a preoperative catheter map or even an intraoperative epicardial map did not modify the increased risk associated with the lack of an intraoperative endocardial map. The inability to complete an intraoperative endocardial map in $52 \%$ of patients is attributed to the low VT induction rate after ventriculotomy and the instability of the induced VT. In patients with severe coronary artery disease, VT often deteriorated into ventricular flutter and fibrillation or was too rapid to allow mapping. Other techniques such as the use of transmural electrodes and the use of an intracavitary balloon electrode array that can allow endocardial mapping before ventriculotomy as reported by others may help overcome this difficulty. ${ }^{6,21}$

The use of cardioplegia was identified as a positive risk factor for postoperative PES-inducible VT and this is likely because of the differences in surgical technique when cardioplegia is required compared with when it is not. When concomitant coronary artery bypass grafting or valve replacement are not needed, cardioplegia is not required and intraoperative endocardial mapping is done followed by subendocardial resection with supplemental cryoablation during normothermic cardiopulmonary bypass in most cases. After this initial mapping and resection, attempts to induce VT are repeated. If VT remains inducible, endocardial mapping and SER/cryoablation are serially repeated until VT can no longer be induced. This method of sequential mapping and resection is based on the finding that VT remains inducible after the initial attempts at map-guided resection in up to $42 \%$ of patients. ${ }^{6,22,23}$ In our series, $10 \%$ of patients had significant left main coronary stenosis and $54 \%$ had severe three-vessel coronary artery disease, which limited the time spent mapping before resection and revascularization. In these cases, the patient was cooled and cardioplegic solution was administered immediately after the completed intraoperative mapping because of concerns of potential myocardial ischemia in this group of patients. In fact, many of these patients with significant coronary artery disease became profoundly ischemic or had ventricular fibrillation after induction of VT, which precluded the completion of intraoperative mapping. After administration of cardioplegic solution and coronary revascularization or valve replacement, or both, attempts to induce VT after resection were not made because of prior experience with increased difficulty in inducing VT after cardioplegia administration. A closer examination comparing those patients who required cardioplegia and those who did not revealed no differences in patient age, aneurysm location, ejection fraction, number of VT morphologies, number of foci, or location of mapped foci. However, univariate analysis did identify a significant difference in the number of patients having completed intraoperative endocardial maps between the two groups $\left(p=0.014, \chi^{2}\right.$ test). It may be that the identification of cardioplegia use as a risk factor for surgical failure in our multivariate analysis is a result of intraoperative mapping biases. With a larger patient population, a multivariate analysis may identify the use of cardioplegia as a dependent variable related to the lack of endocardial mapping. The concerns of myocardial ischemia during repeated cycles of mapping and resection must be balanced against the increased risk of postoperative PES-inducible VT in the absence of sequential mapping and resection. Although only 2 of 48 patients had a perioperative MI, those receiving cardioplegia were identified to be at increased risk of having postoperative PES-inducible VT. 
Our operation-alone success rate of $63 \%$ and those reported by others ${ }^{13-18}$ are lower than the $86 \%$ to $98 \%$ success rates reported by Cox ${ }^{6}$ Hobson and associates, ${ }^{7}$ and Lawrie and associates. ${ }^{10}$ Several differences in technique may explain these differences in outcome. The higher reported percentages ( $46 \%$ to $100 \%$ ) of patients having successful generation of an intraoperative endocardial map may contribute to an improved electrophysiologic outcome. Their high rate of successful intraoperative endocardial mapping be explained by the use of transmural electrodes; the use of isoproterenol, procainamide, or lidocaine to help induce or stabilize VT; or the use of an endocardial balloon electrode array. However, in the series reported by Hargrove and Miller, ${ }^{24}$ intraoperative endocardial maps were completed in $92 \%$ of 269 patients with a success rate similar to our own. In addition to the successful generation of intraoperative endocardial maps, the avoidance of cardioplegia and the use of normothermic bypass during sequential endocardial resection may also explain their outcome data as compared with our own or those of others.

In addition to differences in technique, differences in the patient selection process may likely explain the significant differences in surgical success rates. In the report by Hobson and associates, ${ }^{7}$ only $8 \%$ of patients had an inferior MI with no reported data on mapped foci location; as discussed in that report, the reason for the lower incidence of inferior MI was an institutional bias for patients with an inferior aneurysm and VT with no other disease to have an ICD implantation. In our study if patients with an inferior focus had been selected to have alternative therapies and therefore not included in the series, the operation-alone success rate would increase from $63 \%$ to $83 \%$, a rate similar to that reported by Hobson and associates. ${ }^{7}$ In contrast, the series of Lawrie and associates ${ }^{10}$ involved a patient population in which $41 \%$ had an inferior aneurysm and an inferior wall focus. Nevertheiess, Lawrie and associates ${ }^{10}$ were able to report an operation-alone success rate of $86 \%$ and an overall clinical success rate of $100 \%$. Success after operation for VT appears to be related to surgical and mapping techniques, as well as to patient anatomic selection biases.

Our reported operative mortality rate of $8 \%$ is lower than the operative mortality rate of $17 \%$ previously reported by Garan and associates ${ }^{5}$ from this institution; this mortality difference is significant at the $75 \%$ confidence level $(p=0.24)$. The likely cause of this decrease is our improved patient selection process, which eliminates patients with poor systolic function of the nonaneurysmal ventricular segments, which is associated with higher operative mortality. These patients receive an ICD as an alternative. As a result of this selection bias, only one of the four operative deaths was caused by low-output heart failure, in contrast to results in our earlier series. ${ }^{5}$ The risk factor identified as being a predictor of operative mortality (age 65 years or older) is a risk factor for all patients undergoing cardiac operation and does not appear to be specific for directed ventricular operation. ${ }^{25}$

Although ventricular operation represents an effective method to eliminate VT as a result of ischemic heart disease, placement of an ICD offers only palliative therapy. However, with the identification of risk factors for failure of ventricular operation, the management of those patients possessing these risk factors becomes much more complicated, particularly as ICD technology improves. As ICD technology has improved to include nonthoracotomy lead systems; antitachycardia pacing, which may supplant uncomfortable countershocks; prolonged battery life; and improved memory functions that allow electrogram retrieval and therefore better management of the condition, ${ }^{26}$ the factors involved in the decision between ICD therapy or ventricular operation continue to evolve. Although patients with the risk factors identified by this study have a decreased operation-alone success rate, their overall clinical success rate remains high at $83 \%$. However, ventricular operation poses a higher operative mortality than that for implantation of an ICD; in this study the operative mortality rate was $8 \%$, compared with $1 \%$ to $3 \%$ reported for ICD implantation. ${ }^{27}$ This difference in operative mortality has to be weighed against a 5-year actuarial freedom from recurrent VT of $77.9 \%$ and an actuarial 5-year survival of $75.5 \%$ as shown in this study and a reported 5-year actuarial survival of approximately $60 \%$ for ICD recipients. ${ }^{24} \mathrm{An}$ adjunct for patients having one or more of the identified risk factors for surgical failure is the placement of ICD electrode patches at the time of directed ventricular operation.

\section{REFEREN CES}

1. Ruskin JN, DiMarco JP, Garan H. Out-of-hospital cardiac arrest: electrophysiologic observations and selection of long-term antiarrhythmic therapy. N Engl J Med 1980; 303:607-13.

2. Horowitz LN, Josephson ME, Farshididi A, Spielman SR, Michelson EL, Greenspan AM. Recurrent sustained ventricular tachycardia-3: role of the electrophysiologic study in selection of antiarrhythmic regimens. Circulation 1978;58:986-97.

3. Swerdlow CD, Winkle RA, Mason JW. Determinants of survival in patients with ventricular tachyarrhythmias. N Engl J Med 1983;308:1436-42.

4. Couch OA. Cardiac aneurysm with ventricular tachycardia and subsequent excision of aneurysm. Circulation 1959; 20:251-3.

5. Garan H, Nguyen K, McGovern B, Buckley M, Ruskin 
JN. Perioperative and long-term results after electrophysiologically directed ventricular surgery for recurrent ventricular tachycardia. J Am Coll Cardiol 1986;8:201-9.

6. Cox JL. Patient selection criteria and results of surgery for refractory ischemic ventricular tachycardia. Circulation 1989;79:1163-77.

7. Hobson CE, DiMarco JP, Haines DE, Flanagan TL, Kron IL. The influence of preoperative shock on outcome in sequential endocardial resection for ventricular tachycardia. J THORAC CARdiovasc SuRG 1991;102:348-54.

8. Hargrove WC, Josephson ME, Marchlinski FE, Miller JM. Surgical decisions in the management of sudden cardiac death and malignant ventricular arrhythmias. J THORAC CARDIOVASC SURG 1989;97:923-8.

9. Moran JM, Kehoe RF, Loeb JM, Lichtenthal PR, Sanders JH, Michaelis LL. Extended endocardial resection for the treatment of ventricular tachycardia and ventricular fibrillation. Ann Thorac Surg 1982;34:538-52.

10. Lawrie GM, Pacifico A, Kaushik R, Nahas C, Earle N. Factors predictive of results of direct ablative operations for drug-refractory ventricular tachycardia. J THORAC CARDIOVASC SURG 1991;101:44-55.

11. Josephson ME, Horowitz LN, Spielman SR, Greenspan AM, VandePol C, Harken AH. Comparison of endocardial catheter mapping with intraoperative mapping of ventricular tachycardia. Circulation 1980;61:395-404.

12. Kuchar DL, Ruskin JN, Garan H. Electrocardiographic localization of the site of origin of ventricular tachycardia in patients with prior myocardial infarction. $\mathrm{J}$ Am Coll Cardiol 1989;13:893-900.

13. Hargrove WC, Miller JM, Vassallo JA, Josephson ME. Improved results in the operative management of ventricular tachycardia related to inferior wall infarction. J THORAC CARDIOVASC SuRg 1986;92:726-32.

14. Miller JM, Kienzle MG, Harken AH, Josephson ME. Subendocardial resection for ventricular tachycardia: predictors of surgical success. Circulation 1984;70:624-31.

15. Brandt B, Martins JB, Kienzle MG. Predictors of failure after endocardial resection for sustained ventricular tachycardia. J Thorac Cardiovasc Surg 1988;95:495-500.

16. Swerdlow CD, Mason JW, Stinson EB, Oyer PE, Winkle RA, Derby GC. Results of operations for ventricular tachycardia in 105 patients. J THORAC CARDIOvASC SURG 1986;92:105-13.
17. Saksena S, Hussain SM, Wasty N, Gielchinsky I, Parsonnet $V$. Long-term efficacy of subendocardial resection in refractory ventricular tachycardia: relationship to site of origin. Ann Thorac Surg 1986;42:685-9.

18. Mickleborough LL, Mizuno S, Downar E, Gray GC. Late results of operation for ventricular tachycardia. Ann Thorac Surg 1992;54:832-9.

19. Kron IL, DiMarco JP, Lerman BB, Nolan SP. Resection of scarred papillary muscles improves outcome after surgery for ventricular tachycardia. Ann Surg 1986;203:68590.

20. Ivey TD, Brady GH, Misbach GA, Greene HL. Surgical management of refractory ventricular arrhythmias in patients with prior inferior myocardial infarction. J THORAC CARDIOvasC Surg 1985;89:369-77.

21. Mickleborough LL, Usui A, Downar E, Harris L, Parson I, Gray G. Transatrial balloon technique for activation mapping during operations for recurrent ventricular tachycardia. J THORAC CARDIOVASC SuRg 1990;99:227-33.

22. Kron IL, Lerman BB, Nolan SP, Flanagan TL, Haines DE, DiMarco JP. Sequential endocardial resection for the surgical treatment of refractory ventricular tachycardia. J Thorac Cardiovasc Surg 1987;94:843-7.

23. Haines DE, Lerman BB, Kron IL, DiMarco JP. Surgical ablation of ventricular tachycardia with sequential mapguided subendocardial resection: electrophysiologic assessment and long-term follow-up. Circulation 1988;77:131-41.

24. Hargrove WC, Miller JM. Risk stratification and management of patients with recurrent ventricular tachycardia and other malignant arrhythmias. Circulation 1989;79(Supp1):I178-81.

25. Kirklin JK, Westaby S, Blackstone EH, Kirklin JW, Chenoweth DE, Pacifico AD. Complement and the damaging effects of cardiopulmonary bypass. JTHORAC CARDIOVASC SURG 1983;86:845-57.

26. Mitchell JD, Lee R, Garan H, Ruskin JN, Torchiana DF, Vlahakes GJ. Experience with an implantable tiered therapy device incorporating antitachycardia pacing and cardioverter/defibrillator therapy. J THORAC CARDIOvASC SURG 1993;105:453-63.

27. Gartman DM, Bardy GH, Allen MD, Misbach GA, Ivey TD. Short-term morbidity and mortality of implantation of automatic implantable cardioverter-defibrillator. J THORAC CARdiovasc Surg 1990;100:353-9. 